\section{Beyond relational continuity}

We thank Dr Burch'1 for his interest in our article and agree that the distinction between longitudinal and relational continuity is important.

We are pleased that he too thinks that relational continuity for patients [in] primary care ... should be maximised wherever possible'. He is correct that others have included information and management continuity in the broad concept of continuity of GP care. However, we prefer to separate these and believe that informational continuity is essentially good record-keeping, and management continuity good practice and care plans. Of course, both of these are desirable but our article concerned 'relational continuity'.

The patients' perception of having a deep (trusting) relationship with their GP has been reported by Ridd et al ${ }^{2}$ linked to the number of consultations had with that GP. It shows a linear increase in the depth of the relationship up to eight consultations when there is a $50 \%$ probability of patients thinking they have a 'deep' relationship with the GP concerned. We continue to think relational continuity is by far the most important part of continuity and is the main mechanism generating the important outcomes. ${ }^{3}$ It needs further study in a randomised controlled trial of an intervention to improve continuity.

We do not follow his point about general practice as it is, rather than how we would like it to be', as we very much study general practice as it is. Our earlier report, ${ }^{4}$ in 2019 , reported the measured continuity in a group general practice with 9000 patients over 2 years that was actually received by patients; $65 \%$ of all appointments made by patients aged 65 or over were with their personal GP despite all the GPs being parttime.

Since then, we have learned of other practices where measured continuity of GP care is higher, and we have recently reported how it is also high in two other European countries.

Denis Pereira Gray,

Former GP Partner, Consultant, St Leonard's Practice, Exeter:

Email: denis.pereiragraylabtinternet.com
Kate Sidaway-Lee,

Research Fellow, St Leonard's Practice, Exeter.

Alex Harding,

GP Partner, St Leonard's Practice, Exeter.

Philip H Evans,

Former GP Partner, St Leonard's Practice, Exeter.

\section{REFERENCES}

1. Burch PBM. Beyond relational continuity. [Letter]. Br J Gen Pract 2021; DOI: https://doi.org/10.3399/ bjgp21X716537.

2. Ridd MJ, Lewis G, Peters TJ, Salisbury C. Patientdoctor depth-of-relationship scale: development and validation. Ann Fam Med 2011; 9(6): 538-545.

3. Sidaway-Lee K, Pereira Gray D, Harding A, Evans P. What mechanisms could link GP relational continuity to patient outcomes? Br J Gen Pract 2021; DOI: https://doi.org/10.3399/bjgp21X716093.

4. Sidaway-Lee K, Pereira Gray D, Evans P. A method for measuring continuity of care in dayto-day general practice: a quantitative analysis of appointment data. Br J Gen Pract 2019: DOI: https://doi.org/10.3399/bjgp19X701813.

5. Pereira Gray D, Sidaway-Lee K, Evans P Harding A. Measuring GP continuity at scale. $\mathrm{Br}$ J Gen Pract 2021; DOI: https://doi.org/10.3399/ bjgp21X716129.

DOI: https://doi.org/10.3399/bjgp21X717113

\section{Gadolinium use and risks: an update for colleagues in primary \\ care}

Gadolinium-based contrast agents (GBCA) are widely used in magnetic resonance imaging (MRI) to improve image quality, particularly in angiography and oncologic imaging. ' Gadolinium (Gd) is a naturally occurring heavy metal. In its elemental form, gadolinium is toxic, but, when bound to chelating agents, it is safe for use in humans. GBCA are usually injected intravenously at the start of the MR scan. The extent of gadolinium use in imaging is ubiquitous. In 2018 , it was estimated that, since approval in 1988, over 460 million dosages of gadolinium had been administered worldwide. ${ }^{2}$

Acute allergic reactions to gadolinium are rare. Nephrogenic systemic fibrosis (NSF), a systemic disorder linked to GBCA administration in patients with impaired renal function, ${ }^{3}$ has decreased in incidence with the use of newer GBCAs and a more cautious approach to administration.

The phenomenon of gadolinium deposition in the brain was first described in 2014 on MRI images. ${ }^{4}$ Subsequent studies have confirmed that these signal changes in the brain correspond to gadolinium deposition; this phenomenon occurs in patients with normal renal function. There is no definite evidence linking gadolinium deposition in the brain with any adverse patient outcome.

Despite the lack of causation of a disease state, the action of regulators in the US and Europe has been remarkable. In the US, the Food and Drug Administration took the step of requiring imaging centres to distribute patient Medication Guides to better apprise patients of GBCA-associated risks before administration. The European Medicines Agency went further by restricting the use of certain GBCAs, while concluding that there is currently no evidence that gadolinium deposition in the brain has caused any harm to patients.5

We raise this clinical and regulatory conundrum for the information of colleagues in primary care as, with more direct requesting of a wider range of imaging investigations by GPs, it is likely that questions on this topic will be posed by patients. Colleagues in primary care would be advised to seek up-to-date advice from the radiology department of their local hospital or imaging services provider, as the regulatory position on this topic will undoubtedly alter as new evidence emerges.

Julie Cox,

Consultant Radiologist, South Tyneside and Sunderland NHS Foundation Trust, Sunderland; Visiting Professor, Faculty of Health Sciences and Wellbeing, University of Sunderland, Sunderland.

Email: j.cox1anhs.net 